\title{
An In Vivo Validation of the Application of Acoustic Radiation Force to Enhance the Diagnostic Utility of Molecular Imaging Using 3D Ultrasound
}

\author{
Ryan C. Gessner ${ }^{1,}$, , Jason E. Streeter ${ }^{1,}$, , Roshni Kothadia ${ }^{1}$, Steven Feingold ${ }^{1}$, and Paul A. \\ Dayton ${ }^{1}$ \\ ${ }^{1}$ Joint Department of Biomedical Engineering, University of North Carolina - North Carolina State \\ University
}

\begin{abstract}
For over a decade, the application of acoustic radiation force (ARF) has been proposed as a mechanism to increase ultrasonic molecular imaging (MI) sensitivity in vivo. Presented herein is the first noninvasive in vivo validation of ARF-enhanced MI with an unmodified clinical system. First, an in vitro optical-acoustical setup was used to optimize system parameters and ensure sufficient microbubble translation when exposed to ARF. 3D ARF-enhanced MI was then performed on 7 rat fibrosarcoma tumors using microbubbles targeted to $a_{v} \beta_{3}$ and non-targeted microbubbles. Low-amplitude $(<25 \mathrm{kPa}) 3 \mathrm{D}$ ARF pulse sequences were tested and compared to passive targeting studies in the same animal. Our results demonstrate that a $78 \%$ increase in image intensity from targeted microbubbles can be achieved when using ARF relative to the passive targeting studies. Furthermore, ARF did not significantly increase image contrast when applied to non-targeted agents, suggesting that ARF did not increase non-specific adhesion.
\end{abstract}

\section{Keywords}

microbubble; molecular imaging; targeted imaging; radiation force

\section{Introduction}

\begin{abstract}
Since early observations of acoustic radiation force (ARF) on microbubble contrast agents, researchers have speculated about the utility of this force to push populations of microbubbles in a direction normal to their flow in a patient's vasculature, thereby guiding them out of circulation and against the wall of a vessel (Dayton et al. 1999). It has been hypothesized that increasing ligand-receptor proximity and reducing the velocity of flowing microbubbles would greatly increase the amount of targeted microbubble adhesion in molecular imaging studies (Dayton et al. 2002; Rychak et al. 2007; Rychak et al. 2005; Zhao et al. 2004; Borden et al. 2006). In vitro studies of ARF on microbubbles have demonstrated the ability to increase the quantity of microbubble adhesion over 100 fold compared to
\end{abstract}

(C) 2011 World Federation for Ultrasound in Medicine and Biology. Published by Elsevier Inc. All rights reserved.

Corresponding Author: Paul A. Dayton, Address: 304 Taylor Hall, 109 Mason Farm Road, Chapel Hill, NC, 27599-6136, Phone: (919) 843-9521, Fax: (919) 843-9520, padayton@ bme.unc.edu.

Both authors contributed equally to the generation of this manuscript

Publisher's Disclaimer: This is a PDF file of an unedited manuscript that has been accepted for publication. As a service to our customers we are providing this early version of the manuscript. The manuscript will undergo copyediting, typesetting, and review of the resulting proof before it is published in its final citable form. Please note that during the production process errors may be discovered which could affect the content, and all legal disclaimers that apply to the journal pertain. 
molecular targeting without $\mathrm{ARF}$ (Rychak et al. 2005). In vivo observations performed by intravital microscopy have also demonstrated between four and twenty-fold increases (depending on the vascular environment) in the amount of targeted microbubbles retained in microvasculature after ARF pulses (Rychak et al. 2007). Similarly, with the development of acoustically-active drug delivery vehicles, it has been hypothesized that ARF could be a mechanism to increase vehicle and drug concentration at the desired target site via ultrasound. Researchers have shown the utility of ARF to mediate the concentration of several types of potential therapeutic delivery vehicles (Shortencarier et al. 2004; Lum et al. 2006; Kheirolomoom et al. 2007).

It has also been proposed that ARF might play a role in pulse sequences designed to enhance the detection of targeted contrast agents in molecular imaging. Many current molecular imaging studies involve the use of a waiting period, typically 5-20 minutes, for free (unbound) microbubbles to clear the circulation before imaging of adherent targeted agents can be performed (Lindner et al. 2001). This limitation makes molecular imaging with ultrasound a slow process and likely results in loss of detected signal, as bound microbubbles can detach or degrade over the waiting period. It has been shown that rapid increase in adhesion of targeted microbubbles using ARF can be utilized to help delineate signal from free and bound contrast agents (Patil et al. 2009; Zhao et al. 2007).

However, to date, literature demonstrating the application of ARF in vivo to improve microbubble targeting has been scarce. This is likely due to a disparity between the types of acoustic pulses used for imaging by clinical and preclinical imaging systems, and the acoustic regime over which ARF-induced microbubble displacement is most efficient. Microbubble translation is maximized near the bubble's resonant frequency. For most efficient translation, a large duty cycle is required, and the mechanical index must be low enough to avoid microbubble destruction (Dayton et al. 2002). A long duty cycle and low mechanical index are typically not parameters beneficial for ultrasound imaging, and thus it is likely that the paucity of in vivo ARF-enhanced molecular imaging data is due to the fact that these pulse sequences are not intuitively available on commercial ultrasound equipment.

In this manuscript, we describe the implementation of a widely-used clinical ultrasound system, the imaging parameters of which we have adjusted in order to produce ARF of a magnitude substantial enough to cause non-destructive bubble translation. Following the ARF pulses used to enhance contrast targeting, we utilize contrast-specific imaging pulse sequences for molecular imaging. This study uses a fibrosarcoma tumor model to illustrate the effect of ARF-enhanced molecular imaging compared to conventional passive molecular imaging. Additionally, we investigate the effect on nonspecific adhesion caused by the intentional push of microbubbles away from the center of the lumen toward the distal walls within tumor vasculature. Results from preliminary in vitro studies are also presented which suggest that the pressure of the ARF pulse sequence is a significant factor in promoting lasting bond kinetics between molecularly targeted microbubbles and their target integrins, and that one must balance detrimental over-pushing with ineffective under-pushing to achieve the optimal ARF-enhanced molecular imaging protocol.

\section{Materials and methods}

\section{A. Contrast Agents}

All microbubbles used in our studies were formulated in-house as previously described (Streeter et al. 2010). Briefly, targeted agents were fitted with a cyclic RGD peptide known to bind to $a_{v} \beta_{3}$, an integrin overexpressed on angiogenic endothelium. Likewise, nontargeted microbubbles were produced with a similar lipid formulation, but without the targeting ligand. Both targeted and non-targeted bubbles were of a diameter size distribution 
centered at $1.6 \mu \mathrm{m}$. This was achieved through a differential centrifugation technique (Feshitan et al. 2009).

\section{B. Imaging}

Imaging was performed on a Siemens clinical ultrasound system (Acuson Sequoia 512, Siemens Medical Solutions - Mountain View, CA) using a linear array transducer (model 15L8). The transducer was precisely positioned using a three-axis translational motion stage setup, as previously described (Streeter et al. 2011), and the same probe was used for both imaging and the application of ARF. For volumetric radiation force administration and imaging, the transducer was swept in the elevational direction by a computer controlled motion stage (Model UTS150PP - Newport, Irvine, CA) interfaced through LabView (National Instruments, Austin, TX) to a desktop computer. This motion stage allowed 3D images to be captured and ARF pulses to be delivered uniformly across the volume. ARF pulses were optimized in the pulsed wave (PW) Doppler mode at a frequency of $7 \mathrm{MHz}$. Adjusting the gate size $(18 \mathrm{~mm})$, location of the gate within the field $(65 \mathrm{~mm})$, and blood velocity scale $(1 \mathrm{~m} / \mathrm{sec})$ enabled us to achieve a $25 \%$ duty cycle pulse with a PRF of $25 \mathrm{kHz}$ ( 70 cycles). The amplitude of the ARF pulses was modulated by using the power output dial of the ultrasound system (range: $-7 \mathrm{~dB}$ to $0 \mathrm{~dB}$ ).

\section{Calibration}

Acoustic pressure measurements were determined by a calibrated needle hydrophone (model: HNA-0400, Onda - Sunnyvale, CA) in a water tank. The acoustic pressures produced by the linear array transducer in PW Doppler mode at the previously mentioned parameters were measured at $100 \mu \mathrm{m}$ increments in a $2.2 \times 0.4 \mathrm{~cm}$ area in front of, and parallel to, the aperture using the needle hydrophone. This region of the acoustic pressure field was mapped at $0.5,1,1.5$, and $2 \mathrm{~cm}$ distances from the aperture, for seven different power output settings. The peak negative pressure was measured at each spatial location and power output setting. These pressures were between 8 and $28 \mathrm{kPa}$ for the system output setting studied, and the pressure field deviated by less than $10 \%$ at the locations between 0.5 and $2 \mathrm{~cm}$ from the aperture face (in the water tank).

\section{In vitro studies: effect of ARF pressure}

In order to assess the relationship between output pressure and microbubble translation efficiency, an optical-acoustical system was constructed to enable visualization of microbubble translation during pulsing. A Photron high-speed camera (Model APX-RS San Diego, CA) was fitted with a 60x water immersion objective lens (LUMPlanFI/w, Olympus - Melville, NY) through a single-tube microscope (Edmund Optics, Barrington, $\mathrm{NJ})$. The objective was inserted through a latex seal into the water tank and aligned confocally with and at 90 degrees to the linear array transducer (Figure 1A). A $200 \mu \mathrm{m}$ acoustically and optically transparent cellulose tube (Spectrum Labs, Rancho Dominguez, CA) was placed at the mutual focus, and a fiber optic illuminator was used as a light source for the camera. A dilute concentration of contrast agents $\left(2 \times 10^{7}\right.$ bubbles $/ \mathrm{mL}$; approximately 1:1000 from the initial concentration) was pumped through the tube and ARF pulses were applied perpendicularly to both the flow direction and microbubble buoyancy. Videos were acquired of the contrast agents as they were pushed by the ARF pulses across the diameter of the tube. Data were analyzed offline and the spatial locations of the contrast agents were mapped throughout their translation using a custom-designed program in Matlab (Mathworks - Natick, MA). Bubble motion was tracked at each of the pressure outputs, yielding a relationship between ARF-induced translational bubble velocity and acoustic pressure amplitude. 
A second study was performed in which the axial dimension of the transducer was aligned with the optical axis, and ARF pulses were used to push contrast agents toward the wall of the tube closest to the lens (Figure 1B). The purpose of this study was to visualize the behavior of microbubbles at the tube wall after initial ARF pulses had successfully pushed them to the boundary, and thereby quantify conditions where "over-pushing" occurred. We define over-pushing as any ARF setting that caused microbubble destruction, or lateral microbubble translation along the tube wall. At higher acoustic pressures, lateral motion caused by both secondary radiation forces and force vectors along the curved tube surface in response to primary radiation force would likely cause adherent microbubbles in vivo to be knocked free. Thus, too much radiation force could be detrimental to molecular imaging studies. Videos of the bubbles were acquired for several seconds as they were pushed into the wall by ARF pulses, and analyzed later offline. Data were processed with a pixelwise standard deviation projection through the time axis, thereby enabling the visualization of any lateral movement of bubbles after they initially reached the tube wall.

\section{E. In vitro studies: Estimating optimal in vivo study parameters}

It is known that the amount of ARF experienced by a defined population of microbubbles is a function of the acoustic pressure and the exposure time in the acoustic field. Prior to in vivo studies, we estimated optimal experimental parameters for administering ARF to a volume of tissue in which targeted contrast agents were circulating. We chose to utilize a fixed translational speed of $1 \mathrm{~mm} \cdot \mathrm{s}^{-1}$ for the mechanically scanned transducer, as well as a fixed duty cycle for all ARF administration. The optimal pressure, $\mathrm{P}_{\mathrm{o}}$, was necessarily a balance between efficient bubble translation and bubble-wall interactions. Our goal was to design an experimental protocol in which ARF was applied uniformly across a volume of tissue. Due to the effects of tissue attenuation, $\mathrm{P}_{\mathrm{o}}$ was not constant through the entire depth of tissue. This necessitated a compromise between over-pushing in shallower regions of tissue, and under-pushing at deeper regions of tissue. The optimal initial ARF pressure to maximize targeting efficiency through a specific tumor size was estimated by taking the minimum value of a cumulative error-function (CEF) defined as

$$
C E F=\int_{0}^{D}\left|P_{o}-P(z)\right| d z
$$

where $P(z)$ was the pressure of the radiation force beam derated for attenuation at depth $Z$ into the tumor with an axial diameter $D$. Compensation for tissue attenuation is achieved via the formula

$$
P(z)=P_{i} e^{-\alpha z}
$$

where $a$ is the attenuation coefficient for the tissue (Christensen 1988). A constant tissue attenuation of $0.6 \mathrm{~Np} \cdot \mathrm{cm}^{-1}$ was assumed for the tumors used in the in vivo portion of the study, as this falls within the range of attenuation values previously measured (D'Astous and Foster 1986; Chang et al. 2007). The units of this CEF are in $\mathrm{kPa}$, though the true values returned by the equation are less important than the relative values between different power output settings. We computed the CEF for each of the output settings on the ultrasound system as a method for estimating the optimal ARF pressure amplitude for a given tumor diameter. Similarly, we could estimate the axial distance travelled by microbubbles, $D_{a}$, within a volume of tissue as a result of the ARF pulses by computing 


$$
D_{a}(z)=(\text { velocity }) \cdot(\text { time })=(m \cdot P(z)) \cdot\left(\frac{w_{b}}{v_{T}}\right)
$$

where $m$ is the slope of the curve for velocity of microbubbles as a function of pressure (determined by the in vitro experiments with the schematic seen in Figure 1A, with units $\mathrm{m}$. $\left.(\mathrm{s} \cdot \mathrm{kPa})^{-1}\right), \mathrm{P}(\mathrm{z})$ is the pressure of the ARF beam at depth $z, w_{b}$ is the elevational width of the ARF beam ( $3 \mathrm{~mm}$ ), and $v_{T}$ is the elevational sweep velocity of the transducer during the application of ARF. Thus, the first term in parenthesis will yield the average velocity of the microbubbles exposed to ARF, and the second term will yield the amount of time they are travelling. This calculation assumed an infinite potential path length (i.e. no tube wall to prevent the bubble from being steadily translated).

\section{F. In vivo experiments}

Fischer 344 rats (Charles River Laboratories - Durham, NC) were used for studies of ARFenhanced and conventional molecular imaging (hereafter called "passive targeting" studies, i.e. those not implementing ARF). All animal studies were conducted in accordance with protocols approved by the University of North Carolina School of Medicine's Institutional Animal Care and Use Committee.

Fibrosarcoma tumor models $(\mathrm{N}=8)$ were established from propagated tumor tissue courtesy of the Dewhirst Lab at Duke University. Prior to implantation, rats were anesthetized using isoflurane and their left flank was shaved and disinfected. A $2 \mathrm{~mm}$ incision was made above the quadriceps muscle and a $\sim 1 \mathrm{~mm}^{3}$ piece of tumor tissue was placed subcutaneously. Imaging was performed on tumors after their longest axis had grown to approximately $1 \mathrm{~cm}$.

During the molecular imaging studies, animals were anesthetized with $2.5 \%$ inhaled isoflurane anesthesia mixed with oxygen, and their body temperature was maintained at $37^{\circ} \mathrm{C}$ through the use of a temperature-controlled heating pad. The area to be imaged was shaved, further depilated using a chemical hair remover, and then coupled to the ultrasound transducer using gel as previously described (Streeter et al. 2011).

Microbubbles were administered in bolus injections via a 24-gauge tail vein catheter. The concentrations of stock solutions for both the targeted and control bubbles were measured prior to each imaging study using a particle sizer as previously described (Streeter et al. 2010). The appropriate volume of stock solution was added to the catheter via a micropipette tip and flushed with $100 \mu \mathrm{L}$ of sterile saline such that a contrast dose of $2 \times 10^{8}$ bubbles was administered consistently. Because of the dose limitations with the injected volume of saline and contrast into the animals, two different types of studies were performed over several days to examine (a) the effect of ARF pulse amplitude on microbubble targeting efficiency relative to passive targeting, and (b) the effect of ARF at promoting non-specific adhesion in control bubbles relative to targeted bubbles also exposed to ARF.

For study type (a), each animal underwent four distinct injection and ARF protocols in the following order: (1) passive targeting (no application of ARF) using targeted microbubbles (2) application of ARF with a pressure of $4 \mathrm{kPa}$ using targeted microbubbles (3) application of ARF using a pressure of $13 \mathrm{kPa}$ with targeted microbubbles (4) application of ARF with a pressure of $21 \mathrm{kPa}$ using targeted microbubbles. After each of these four imaging protocols, there was a minimum wait time of 10 minutes to ensure that there were no circulating microbubbles remaining. Similarly for study type (b), each animal underwent distinct injection and ARF protocols in the following order: (1) passive targeting with control microbubbles (2) application of ARF with a pressure of $13 \mathrm{kPa}$ with control microbubbles (3) passive targeting with targeted microbubbles (4) application of ARF with a pressure of 
$13 \mathrm{kPa}$ with targeted microbubbles. Between each type (b) experiment, there was a minimum wait time of 10 minutes to ensure that there were no circulating microbubbles remaining in the tumor vasculature. Due to injection volume limitations, study types (a) and (b) were not performed in the same animal on the same day. In order to compare type (a) and type (b) experiments, data were normalized to the mean image intensity within the volumetric passive targeting image data (using targeted microbubbles without application of ARF) in each animal because this imaging protocol occurred in both type (a) and type (b) studies.

During all ARF-enhanced targeting studies, radiation force pulses were applied over the entire volume of the tumor using the mechanically scanned transducer at pressures of 4, 13, or $21 \mathrm{kPa}$ (number of animals imaged at each setting: $4 \mathrm{kPa}: \mathrm{N}=5,13 \mathrm{kPa}: \mathrm{N}=7,21 \mathrm{kPa}$ : $\mathrm{N}=5$ ). The transducer was swept at a constant speed of $1 \mathrm{~mm} / \mathrm{s}$ across the tumor volume after contrast agents were administered to the animal, for a total of ten passes following the injection. During passive targeting studies, ARF pulses were not administered $(\mathrm{N}=4)$. After waiting approximately 10 minutes for freely-circulating bubbles to clear from the animal's system, a 3D imaging scan was acquired across the tumor with inter-plane step sizes of 400 $\mu \mathrm{m}$ in the ultrasound system's Cadence contrast imaging mode (CPS). The bound microbubbles were then destroyed using a high mechanical index b-mode 3D scan, and then the tumor reimaged in CPS mode at the same slice locations for a baseline measurement of no targeted agents.

Our inter-image step-size was below the $-6 \mathrm{~dB}$ elevational beam width determined in a previous study (Feingold et al. 2010) to be $\sim 800 \mu \mathrm{m}$. Thus the volumetric data was downsampled offline at $800 \mu \mathrm{m}$ intervals to yield independent imaging frames for analysis as previously described (Streeter et al. 2010). The mean pixel intensities within the background-subtracted images were computed, producing a mean targeted bubble intensity for every $2 \mathrm{D}$ image plane within the $3 \mathrm{D}$ image acquisition. As previously mentioned, the mean targeted microbubble intensity values were normalized to the mean of the passive targeting microbubble injection. This allowed us to reduce the impact of inter-animal tumor heterogeneity when making comparisons between animals. The brightness of adherent MCAs was assumed to be correlated with the degree of biomarker expression. Thus, throughout the remainder of this manuscript, "mean pixel intensity" will refer to the quantity defined by the difference between the average image's intensity before the destruction pulse and the average intensity of the image for the same region of interest after the destruction pulse. This "mean pixel intensity" metric is a way to approximate the quantity of adherent microbubbles at a single 2D image slice's location. The statistical significance of our comparisons between the mean microbubble targeting measured by the different imaging study settings was assessed by using Matlab to implement a two-sided Student's t-test assuming equal variances. Significance between two different distributions were considered at a value of $\mathrm{p}<0.05$.

\section{Results}

\section{A. In vitro studies: effect of ARF pressure}

The positions of multiple bubbles $(4 \leq \mathrm{N} \leq 10)$ were tracked through space and time and a linear relationship $\left(\mathrm{R}^{2}=0.953\right)$ between velocity and radiation force amplitude was observed, with a slope of $5.018 \mu \mathrm{m} \cdot(\mathrm{s} \cdot \mathrm{kPa})^{-1}$ (Figure 2A). For this linear curve fit, the yintercept was set to 0 (corresponding with a velocity of zero for microbubbles not exposed to ARF).

At each acoustic pressure setting tested between 8 and $28 \mathrm{kPa}$, the ARF pulses were able to force the microbubbles out of the center of the cellulose tube and against the wall. As the 
pressure was increased, the aggressiveness of the lateral bubble movement and aggregation due to secondary radiation force increased (Figure 2B). Based on qualitative observations from pixelwise time-axis standard deviation projections (Figure 2B), it was hypothesized that the secondary radiation force at pressures greater than $10 \mathrm{kPa}$ would not facilitate lasting ligand-integrin bond kinetics, since ARF pressures above this threshold caused bubbles to laterally translate along the cellulose tube wall. Thus, $\mathrm{P}_{\mathrm{O}}$ was estimated to be 10 $\mathrm{kPa}$, as this setting would provide the best compromise between efficient bubble translation $(\sim 50 \mu \mathrm{m} / \mathrm{s})$ and minimization of lateral movement after contact with the vessel wall.

\section{B. In vitro studies: Estimating optimal in vivo study parameters}

The CEF computations demonstrated that there was no single pressure appropriate for all tumor sizes. A 2D surface plot of the CEF values illustrates that for varying tumor sizes, there exist different acoustic pressures that will optimize radiation-force enhanced targeting over the entire tumor depth (Figure 3A). The CEF predicted that the $13 \mathrm{kPa}$ pressure setting would be the most effective in vivo protocol for a $1 \mathrm{~cm}$ tumor. A $4 \mathrm{kPa}$ and a $21 \mathrm{kPa}$ setting were also tested in vivo to compare the relative targeting efficiencies to the $13 \mathrm{kPa}$ setting predicted by the CEF model.

The calculations of $\mathrm{D}_{\mathrm{a}}$, the expected distance microbubbles would translate in vivo along the ARF beam's propagation axis, revealed that both the initial push pressure and the depth into tissue determined whether a contrast agent would be sufficiently pushed out of circulation to the luminal wall (Figure 3B). In a previous histological analysis of vessels within xenografted tumors, $100 \mu \mathrm{m}$ was the upper bound on the range of vessel diameters (Palmowski et al. 2008). It was predicted that the three pressure settings tested in vivo would provide sufficient microbubble translation $\left(\mathrm{D}_{\mathrm{a}} \geq 50 \mu \mathrm{m}\right)$ at tissue depths less than $3.1 \mathrm{~cm}$, $2.4 \mathrm{~cm}$, and $0.5 \mathrm{~cm}$ for the $21 \mathrm{kPa}, 13 \mathrm{kPa}$ and $4 \mathrm{kPa}$ ARF amplitudes respectively.

\section{In vivo results}

Microbubbles targeted to angiogenic vasculature were used to compare ARF-enhanced targeting to conventional passive targeting (Figure 4). Of the tested ARF pulsing schemes, the maximum increase in microbubble targeting, as measured by mean pixel intensities, was achieved using ARF pulses with a $13 \mathrm{kPa}$ amplitude and a cRGD targeted bubble (nomenclature: "RF-13/cRGD ${ }^{+*}$ ). In all of the animals that were tested, the volumetric targeted intensity was greater using RF-13/cRGD ${ }^{+}$than with passive targeting (nomenclature: "RF-0/cRGD ${ }^{+\prime}$ ). Notably, there was improved microbubble retention in $91 \%$ of all 2D image slices of RF-13/cRGD ${ }^{+}$data collected when compared to the RF-0/cRGD ${ }^{+}$ studies (Table 1). In the remaining $9 \%$ of 2D image slices (in which the passive targeting images were brighter than the RF-13/cRGD ${ }^{+}$images), there was less than $1 \mathrm{~dB}$ difference between the mean pixel intensity of RF-13/cRGD ${ }^{+}$data as compared to the RF-0/cRGD ${ }^{+}$ case. Also of note, of the $9 \%$ of $2 \mathrm{D}$ image slices in which RF-13/cRGD+ was not as bright as the passive targeting run, $80 \%$ of these were within the same animal. This was a likely an indication of a less angiogenic and more necrotic tumor in this one animal, and thus there was very little adhesion of targeted agents, either with or without ARF.

The increase in mean pixel intensity across all animals was $77.8 \%$ higher with the RF-13/ $\mathrm{cRGD}^{+}$over the RF-0/cRGD ${ }^{+}$case: $11.4 \pm 7.0$, compared to $6.4 \pm 4.8$ respectively. The values represent baseline-subtracted pixel intensities, and thus the units are $\mathrm{dB}$. The error in these values is the standard deviation of the baseline-subtracted pixel intensities. Similarly, both the RF-4 $(8.8 \pm 7.9)$ and RF-21 $(8.8 \pm 7.0)$ settings produced a statistically significant increase in targeting of $\mathrm{CRGD}^{+}$bubbles relative to the RF-0/cRGD ${ }^{+}$case. Of note, the RF-13/cRGD ${ }^{-}$case suggested a slight increase in mean pixel intensity for non-specific microbubble adhesion relative to the $\mathrm{RF}-0 / \mathrm{CRGD}^{-}$case, however differences were not 
significant $(\mathrm{p}=0.15)$. The histograms created from the amalgamation of all unnormalized data show that, in general, the mean pixel intensity was higher for both ARF-enhanced and passive targeting studies (Figure 4B) compared to non-targeted controls. Furthermore, these histograms show that the RF-13/cRGD ${ }^{+}$studies produced the greatest number of enhanced pixels. Figure 5 illustrates an example of ARF-enhanced targeted imaging and conventional passive targeting. In this image, the tumor volume is presented with a conically-stratified hinged cutaway, wherein elliptical regions of interest were drawn such that they did not follow the tumor border but instead cut into the interior of the tumor. This allows for microbubble targeting from within the tumor margins to be visualized on the rendered 3D surface, which we found was useful in visualizing 3D molecular imaging data.

\section{Discussion}

\section{A. Optimizing radiation force: in vitro predictions and in vivo results}

Using ARF to facilitate molecular targeting of microbubbles to integrins expressed on diseased endothelium is an intuitive approach to improving the sensitivity of this diagnostic imaging methodology, but its application is non-trivial. Our in vitro studies allowed us to observe the behavior of microbubbles exposed to ARF pulses both during their translation and after making contact with the wall of a vessel-mimicking phantom. These analyses allowed us to tune parameters to predict the optimal settings for in vivo studies.

The calculation of the CEF demonstrated that because of the effects of tissue attenuation, there is no single setting for ARF amplitude that is universally appropriate to increase molecular imaging sensitivity at all tissue depths. Our CEF model predicted that ARF pulses with $13 \mathrm{kPa}$ pressure amplitude to be the most effective of the three amplitudes studied. The model suggestions were supported by the in vivo study, as the $13 \mathrm{kPa}$ pulses had the highest microbubble targeting efficiency. The CEF predictions for the two other pressure amplitudes tested (4 and $21 \mathrm{kPa}$ ) were very similar (Figure 3), which was also reflected in the in vivo data, as these pressures resulted in similar increases in microbubble targeting, and were both less effective than the $13 \mathrm{kPa}$ ARF pulses. This CEF model assumed a constant attenuation of $0.6 \mathrm{~Np} \cdot \mathrm{cm}^{-1}$ throughout the depth of the tumor, which did not take into account the attenuation provided by the scatter of microbubbles in the tissue. Moreover, our method of tuning ARF pressure via the CEF assumes that a uniform density of integrin (or other biomarker) expression exists throughout the entire tumor volume. While this is likely not true in the highly heterogeneous microenvironment of tumors, unless the heterogeneity is known a priori, the best strategy for improving molecular targeting is one which maximizes the probability of microbubble bond formation at all locations within the tumor.

Our in vivo studies demonstrated an average increase in microbubble targeting of nearly $80 \%$ relative to traditional passive targeting studies, as measured by average pixel intensities within the baseline-subtracted images. It is important to emphasize that $91 \%$ of all the $2 \mathrm{D}$ image slices within the ARF-enhanced datasets exhibited greater microbubble targeting than the corresponding image frames taken from the passive targeting data. Of the remaining slices that exhibited greater microbubble adhesion in the passive targeting case, most were outliers found in a single animal that showed poor microbubble targeting in all experiments. This, along with the rest of the data summarized in Table 1, makes a compelling argument that ARF can improve the diagnostic utility of ultrasonic molecular imaging studies.

When comparing the control microbubbles (non-targeted) to the cyclic RGD microbubbles (targeted) in the absence of ARF, it was apparent that non-specific targeting was observed for the microbubble concentrations and tumor models used in this study. However, the application of ARF significantly increased the mean pixel intensity from the targeted agents, without significantly increasing the mean pixel intensity from the control agents - thereby 
providing a means to increase signal to noise in situations where there is a high non-specific targeting background.

A persistent question surrounding the usefulness and applicability of ARF to in vivo molecular imaging studies is whether pushing populations of bubbles away from the proximal walls of vessels could actually be detrimental to maximizing the number of microbubble-integrin bonds. While microbubble targeting on proximal vessel walls is likely negligible during the application of ARF pulses, after the pulses stop, these binding sites are free to be populated by the passive targeting of bubbles circulating through the vasculature.

Regardless, based on our in vitro observations, the amount of increased bubble adhesion due to ARF substantially outweighs any loss of targeting on the proximal vessel walls. Another concern is whether acoustically forcing bubbles into vessel walls will cause an increase in non-specific adhesion and thereby increase the rate of false-positive diagnoses. In this study, there was no significant difference in targeting efficiency between the control bubble with ARF application compared to the control bubble with no ARF (Figure 4). However, we suggest that this would need to be re-evaluated for different adhesion ligands and tumor types.

\section{B. Comparison to previous work}

Previous in vitro work studying the increase in targeting efficiency of ARF-enhanced studies relative to passive targeted bubbles adhesion has demonstrated a large dependence on microbubble concentration. There was approximately 3 times more adhesion in passive targeting experiments without an appreciable difference in ARF efficiency when the bubble concentration was increased from $2.5 \times 10^{6}$ to $25 \times 10^{6}$ bubbles $/ \mathrm{mL}$ (Rychak et al. 2005). This suggests that a larger difference in microbubble targeting effectiveness will be apparent if a lower concentration of microbubbles is used, though these studies have yet to be performed in vivo. Additionally, since the magnitude of radiation force is highest at the resonant frequency of a microbubble (Dayton et al. 2002), the frequency of our radiation force pulses could have been a source of reduction in expected targeting effectiveness. We were pulsing at $7 \mathrm{MHz}$, which was slightly higher than the resonance frequency of our bubbles ( $\sim 6 \mathrm{MHz}$, as calculated using the method described in (Quaia 2005)). Finally, secondary radiation force is known to create aggregates of multiple microbubbles after contacting vessel walls (Dayton et al. 1999; Rychak et al. 2005; Kotopoulis and Postema 2009) (Figure 2B). If these aggregates form lasting bonds with integrin targets, they will contribute to the image signal intensity differently than a single bubble would. This could have contributed to the variability observed in our in vivo studies.

In prior in vivo molecular imaging studies implementing ARF, Rychak et al. (2007) optically observed a $>20$ fold increase in microbubble adhesion in vivo using intravital microscopy in both the femoral artery and vein of a mouse model for inflammation, although this study relied on optically counting microbubbles through invasive intravital microscopy to quantify retention rather than using ultrasound imaging. Thus, a direct correlation between Rychak's data and the data presented herein is not possible. Gessner et al. (2009) previously demonstrated the administration of ARF and acoustic detection of the targeted bubbles using a prototype high-resolution probe and custom pulse sequences, with an average microbubble targeting improvement of $13 \mathrm{~dB}$ across all image slices when ARF was used compared to passive targeting. There were many differences between that study and this one, including the ARF parameters, and the imaging techniques' contrast sensitivities, which could account for the differences between the reported increase in sensitivity facilitated by radiation force in that paper $(13 \mathrm{~dB} \approx 4.5$ fold increase in detected contrast signal) relative to data presented here. 


\section{Limitations and future directions}

It was beyond the scope of the in vitro study of observations of contrast agents interacting with the tube wall to quantify the motion and trajectories of the bubbles after contacting the tube wall. This was because the aggregates of multiple contrast agents continually formed clusters of bubbles in an erratic fashion due to secondary radiation forces, and thus made tracking individual contrast agents very difficult. The lack of this analysis notwithstanding, the standard deviation projections seen in Figure 2B demonstrate this effect and suggested that higher pressures would reduce the likelihood of lasting ligand-integrin bond formations. A more thorough analysis of this lateral motion would help define $\mathrm{P}_{\mathrm{o}}$ more accurately than our coarse ability to adjust the amplitude of the ARF pulses permitted.

As mentioned previously, the best strategy for improving targeting is one that maximizes the probability of microbubble adhesion throughout the tumor. Due to attenuation effects, it was not possible in our in vivo studies to apply ARF uniformly at all axial depths. One potential strategy to apply a more homogeneous ARF profile to multiple depths within tissue (not examined in these studies) would be to sweep through multiple pressures at each spatial location. If at first a high pressure is used, it facilitates microbubble targeting deeper into tissue (due to attenuation) while simultaneously over-pushing at shallow depths. If the pressure is steadily reduced, microbubbles at shallower depths will be pushed at a more appropriate pressure without negatively affecting the targeted bubbles deeper in the tissue, since the pressure at these depths will have been reduced and thus the secondary radiation force effects minimized. Of note, over-pushing targeted contrast agents would likely only be detrimental to molecular imaging studies in which the lasting integrity of the microbubble's bond is critical. This limitation would probably not be a concern in ARF-mediated drug delivery, where the ultimate goal is rapid and efficient localization and release of a microbubble's payload. In many ultrasound-mediated therapies, the microbubbles are disrupted immediately after pushing towards the endothelium, and long-term adhesion is not required (Shortencarier et el. 2004; Lum et al. 2006; Kheirolomoom et al. 2007).

The time window over which radiation force is applied may also affect the increase in microbubble adhesion; if radiation force is administered continuously over several minutes as microbubbles circulate through the animal, more bubbles will be pushed against the endothelium, though this increased microbubble traffic at the vessel wall could be counterproductive if it causes previously adherent agents to become dislodged.

The experiments performed in this manuscript were done on a widely used clinical scanner without any additional customization of the software or pulse sequences, although we suggest that improved results would be obtained if dedicated optimized pulse sequencing and beam focusing/defocusing were available. Our approach did rely on mechnanical scanning of the transducer to uniformly apply ARF pulses from the 1D linear array across the volume of tumor tissue, though this would be unnecessary if a 2D matrix array probe were available for high-resolution contrast imaging.

\section{Conclusion}

The studies presented herein provide validation of acoustic radiation force applied with a clinical ultrasound system as a mechanism to enhance molecular imaging. In all in vivo studies, the average targeted microbubble signal throughout the 3D tumor volume was greater when using ARF (at $13 \mathrm{kPa}$ ) when compared with passive targeting studies. Our 3D imaging results demonstrate an improvement in signal intensity of $77.8 \%$ to our molecular target over conventional passive molecular imaging without an increase in non-specific targeting. Furthermore, experiments suggest that for volumetric administration of radiation force, there exists an optimal pressure for ARF-enhanced targeting, which is a compromise 
between efficient bubble translation and adhesion-disruptive secondary radiation force and lateral translation effects.

\section{Acknowledgments}

The authors appreciate the assistance of Ismayil Guracar, from Siemens Medical Solutions, in optimizing parameters to produce acoustic radiation force on the Siemens clinical ultrasound system. The efforts of James Tsuruta in cRGD lipid conjugation are also appreciated. Funding was provided by NIH R01 EB009066, NIH R01 EB008733, and the University Cancer Research Fund.

\section{References}

Borden MA, Sarantos MR, Stieger SM, Simon SI, Ferrara KW, Dayton PA. Ultrasound radiation force modulates ligand availability on targeted contrast agents. Mol Imaging. 2006; 5:139-47. [PubMed: 16954028]

Christensen, DA. Ultrasonic Bioinstrumentation. John Wiley \& Sons; 1988.

Chang CH, Huang SW, Yang HC, Chou YH. Reconstruction of Ultrasonic Sound Velocity and Attenuation Coefficient Using Linear Arrays: Clinical Assessment. Ultrasound Med Biol. 2007; 33:1681-87. [PubMed: 17629607]

D’Astous FT, Foster FS. Frequency Dependence of Ultrasound Attenuation and Backscatter in Breast Tissue. Ultrasound Med Biol. 1986; 12:795-808. [PubMed: 3541334]

Dayton PA, Allen JS, Ferrara KW. The magnitude of radiation force on ultrasound contrast agents. J Acoust Soc Am. 2002; 112:2183-92. [PubMed: 12430830]

Dayton P, Klibanov A, Brandenburger G, Ferrara K. Acoustic radiation force in vivo: a mechanism to assist targeting of microbubbles. Ultrasound Med Biol. 1999; 25:1195-201. [PubMed: 10576262]

Feingold S, Gessner R, Guracar IM, Dayton PA. Quantitative volumetric perfusion mapping of the microvasculature using contrast ultrasound. Invest Radiol. 2010; 45:669-74. [PubMed: 20808232]

Feshitan JA, Chen CC, Kwan JJ, Borden MA. Microbubble size isolation by differential centrifugation. Journal of Colloid and Interface Science. 2009; 329:316-24. [PubMed: 18950786]

Gessner, R.; Lukacs, M.; Lee, M.; Tsuruta, J.; Foster, FS.; Dayton, PA. Radiation force-enhanced targeted imaging and near real-time molecular imaging using a dual-frequency high-resolution transducer: In-vitro and in-vivo results. International IEEE Ultrasonics Symposium (IUS); 2009. p. 9-12.

Kheirolomoom A, Dayton PA, Lum AF, Little E, Paoli EE, Zheng H, Ferrara KW. Acoustically-active microbubbles conjugated to liposomes: characterization of a proposed drug delivery vehicle. $\mathrm{J}$ Control Release. 2007; 118:275-84. [PubMed: 17300849]

Kotopoulis S, Postema M. Microfoam formation in a capillary. Ultrasonics. 2009; 50:260-68. [PubMed: 19875143]

Lindner JR, Song J, Christiansen J, Klibanov AL, Xu F, Ley K. Ultrasound assessment of inflammation and renal tissue injury with microbubbles targeted to P-selectin. Circulation. 2001; 104:2107-12. [PubMed: 11673354]

Lum AF, Borden MA, Dayton PA, Kruse DE, Simon SI, Ferrara KW. Ultrasound radiation force enables targeted deposition of model drug carriers loaded on microbubbles. J Control Release. 2006; 111:128-34. [PubMed: 16380187]

Palmowski M, Huppert J, Hauff P, Reinhardt M, Schreiner K, Socher MA, Hallscheidt P, Kauffmann GW, Semmler W, Kiessling F. Vessel fractions in tumor xenografts depicted by flow- or contrastsensitive three-dimensional high-frequency Doppler ultrasound respond differently to antiangiogenic treatment. Cancer Res. 2008; 68:7042-49. [PubMed: 18757418]

Patil AV, Rychak JJ, Allen JS, Klibanov AL, Hossack JA. Dual frequency method for simultaneous translation and real-time imaging of ultrasound contrast agents within large blood vessels. Ultrasound in Medicine and Biology. 2009; 35:2021-30. [PubMed: 19828229]

Quaia, E. Contrast media in ultrasonography_basic principles and clinical applications. New York: Springer; 2005. 
Rychak JJ, Klibanov AL, Hossack JA. Acoustic radiation force enhances targeted delivery of ultrasound contrast microbubbles: in vitro verification. IEEE Trans Ultrason Ferroelectr Freq Control. 2005; 52:421-33. [PubMed: 15857050]

Rychak JJ, Klibanov AL, Ley KF, Hossack JA. Enhanced targeting of ultrasound contrast agents using acoustic radiation force. Ultrasound Med Biol. 2007; 33:1132-9. [PubMed: 17445966]

Shortencarier MJ, Dayton PA, Bloch SH, Schumann PA, Matsunaga TO, Ferrara KW. A method for radiation-force localized drug delivery using gas-filled lipospheres. IEEE Trans Ultrason Ferroelectr Freq Control. 2004; 51:822-31. [PubMed: 15301001]

Streeter JE, Gessner R, Miles I, Dayton PA. Improving sensitivity in ultrasound molecular imaging by tailoring contrast agent size distribution: in vivo studies. Mol Imaging. 2010; 9:87-95. [PubMed: 20236606]

Streeter JE, Gessner RC, Tsuruta J, Feingold S, Dayton PA. Assessment of Molecular Imaging of Angiogenesis with Three-Dimensional Ultrasonography. Mol Imaging. 2011; 10:460-8. [PubMed: 22201537]

Zhao S, Borden M, Bloch SH, Kruse D, Ferrara KW, Dayton PA. Radiation-force assisted targeting facilitates ultrasonic molecular imaging. Mol Imaging. 2004; 3:135-48. [PubMed: 15530249]

Zhao S, Kruse DE, Ferrara KW, Dayton PA. Selective imaging of adherent targeted ultrasound contrast agents. Phys Med Biol. 2007; 52:2055-72. [PubMed: 17404455] 


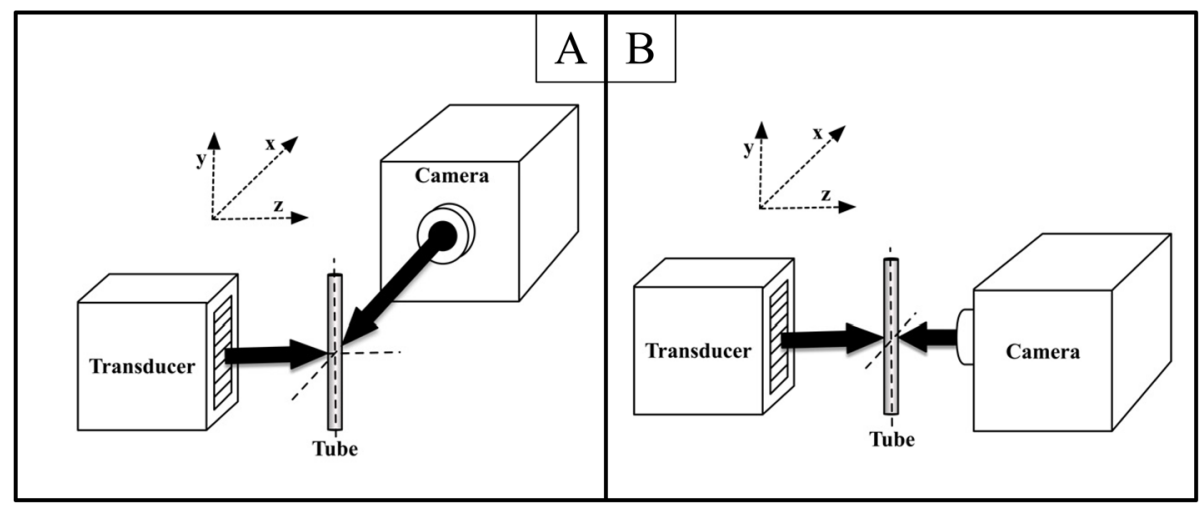

Figure 1.

Experimental diagrams for the in vitro characterization of the effects of radiation force on microbubbles. A: System used to determine the relationship between microbubble translational velocity and output pressure from the transducer. B: System used to examine the effects of secondary radiation force on contrast agents after making contact with the tube wall. In panel $\mathrm{A}$, the optical axis is along the $\mathrm{x}$ direction, the acoustic axis is along the $\mathrm{z}$ direction and the microbubble flow axis is along the $y$ direction. Similarly, in panel $\mathrm{B}$, the optical axis and the acoustic axis are along the $\mathrm{z}$ direction and the microbubble flow axis is along the y direction. 


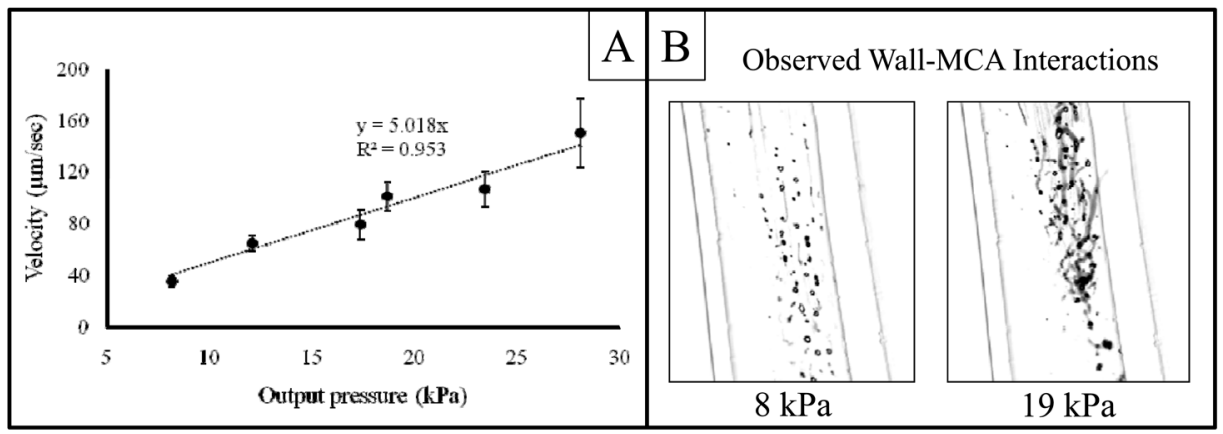

Figure 2.

Results from optical observations of in vitro radiation force on microbubbles. A: The translational velocity of contrast agents as a function of radiation force pressure amplitude. $\mathrm{B}$ : Images of standard deviation projections of microbubble lateral translation along the tube wall during radiation force pulsing at high $(19 \mathrm{kPa})$ and low $(8 \mathrm{kPa})$ pressures. As the pressure is increased, lateral motion along the wall caused by secondary radiation force can be clearly visualized as non-spherical trails. Note: the displayed images are negatives of the actual projections - large values appear dark in these images. 


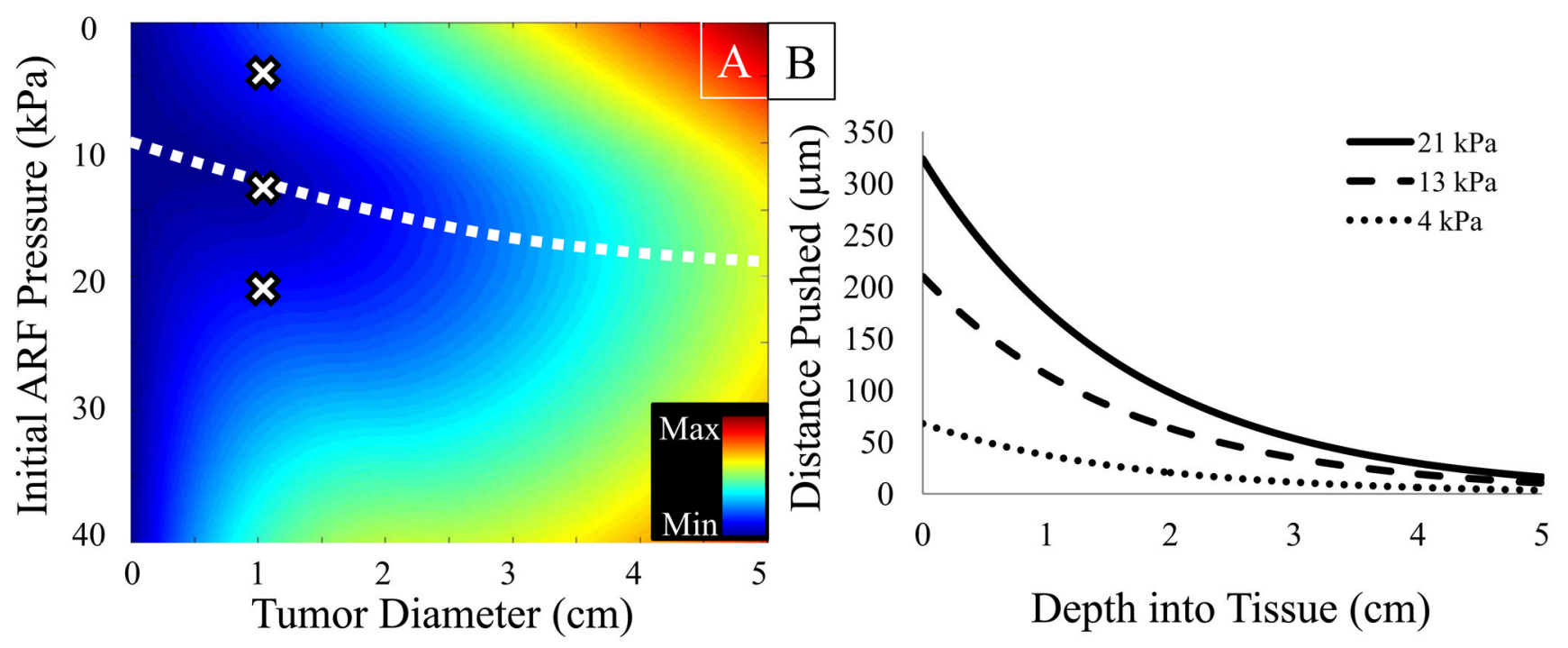

Figure 3.

A: The CEF plotted as a function of initial ARF push pressure and tumor diameter. Minimizing the $\mathrm{CEF}$ at a given tumor diameter provides the basis for estimating the optimal push protocol for an in vivo study (white dashed line). The X's illustrate the three output settings that were tested and compared in the in vivo validation studies. B: Simulations predicting the distance a microbubble would be pushed by ARF pulses for the three different initial pressure amplitudes as a function of depth into tissue. 


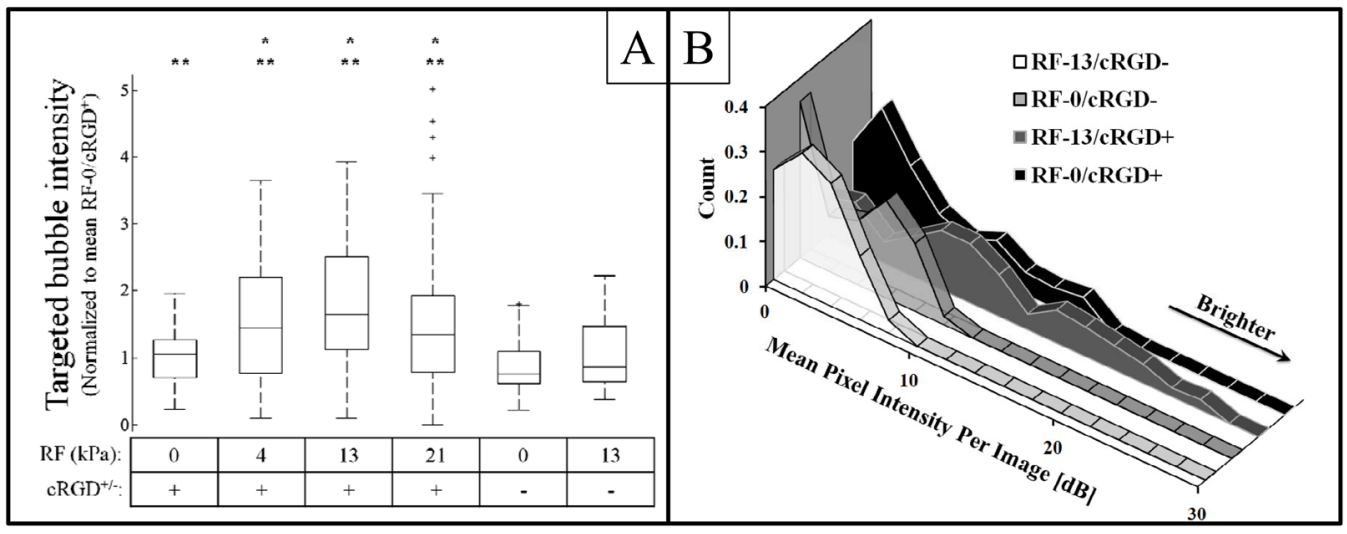

Figure 4.

A: A box and whiskers plot displaying the distribution of all mean intensities within baseline-subtracted targeted bubble images collected in this study. Data is normalized to the mean pixel intensity within the $0 \mathrm{kPa} / \mathrm{cRGD}+$ dataset collected in each study. Asterisks indicate a statistically significant increase in targeting $(\mathrm{p}<0.05)$ over: * - the RF-0/cRGD+ data, ** - the RF-0/cRGD- data. B: A comparison of the histograms of pixel intensities for all image slices used in studies using control and targeted microbubbles with and without radiation force at $13 \mathrm{kPa}$. These histograms are area-normalized (not intensity normalized), and show a larger proportion of bright pixel values when targeted bubbles are used, and even more when radiation force is used with targeted bubbles. 


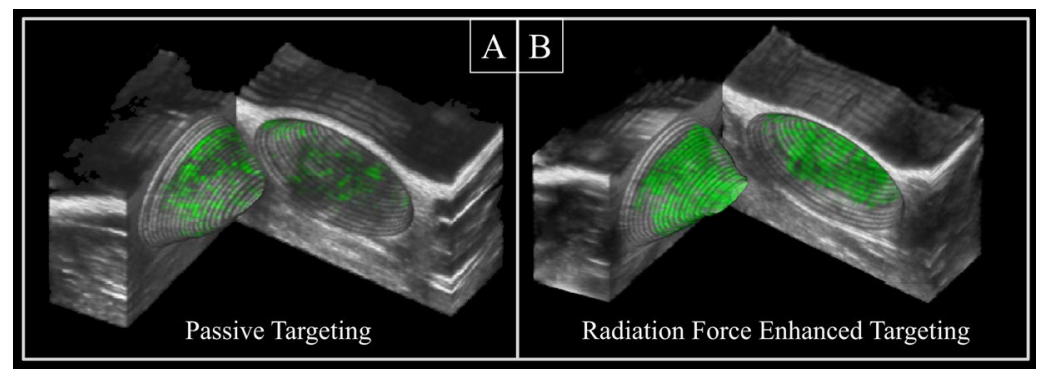

Figure 5.

A side-by-side comparison of two 3D conically-stratified hinged cutaway images. B-mode images (grayscale) are registered to the corresponding CPS images acquired 10 minutes after contrast injection (green). In both of these images, the same dose of targeted microbubbles was administered to the same animal. Radiation force was not applied in image $\mathrm{A}$, and was applied at $13 \mathrm{kPa}$ in image $\mathrm{B}$. The imaging field of view was $1.2 \mathrm{~cm} \times 1.1$ $\mathrm{cm} \times 1.2 \mathrm{~cm}$. 


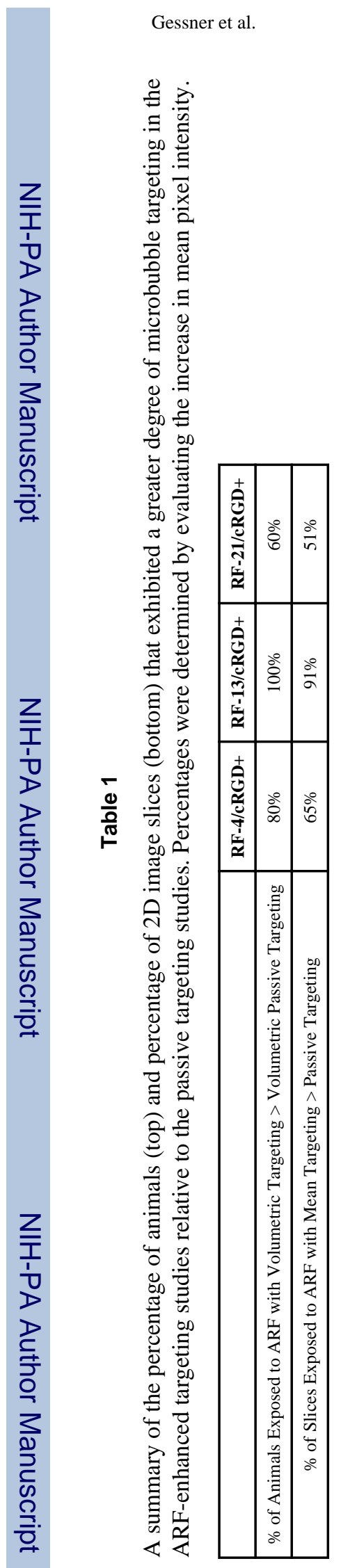

Ultrasound Med Biol. Author manuscript; available in PMC 2013 April 01. 